\title{
Enquête
}

Archives de la revue Enquête

5 | 1989

Biographie et cycle de vie

\section{Structures mémorielles, littérature et biographie}

\section{Régine Robin}

\section{(2) OpenEdition}

\section{Journals}

Édition électronique

URL : http://journals.openedition.org/enquete/116

DOI : 10.4000/enquete. 116

ISSN : 1953-809X

\section{Éditeur :}

Cercom, Éditions Parenthèses

\section{Édition imprimée}

Date de publication : 2 mars 1989

\section{Référence électronique}

Régine Robin, «Structures mémorielles, littérature et biographie », Enquête [En ligne], 5 | 1989, mis en ligne le 27 juin 2013, consulté le 19 avril 2019. URL : http://journals.openedition.org/enquete/116 ; DOI : 10.4000/enquete. 116

Ce document a été généré automatiquement le 19 avril 2019. 


\title{
Structures mémorielles, littérature et biographie
}

\author{
Régine Robin
}

\section{Le passé fixé}

$1 \quad$ Mes deux notions sont problématiques.

2 Malgré un grand livre de Maurice Halbwachs, malgré les recherches de ses continuateurs et les nombreux commentaires que la notion de "mémoire collective » a suscités ${ }^{1}$, nous n'avons pas à ce jour une définition satisfaisante. Nous dirons simplement pour donner un cadre à notre réflexion que nous engloberons par là tout le domaine de l'appropriation sociale du passé, de la rétrospective collective, de la gestion, de la régie de ce passé. Il faudra bien entendu mettre de l'ordre là-dedans et peut-être abandonner une vision aussi englobante du phénomène.

Quant au « roman familial », la notion vient de Freud et désigne selon J. Laplanche et J.B. Pontalis « des fantasmes par lesquels le sujet modifie imaginairement ses liens avec ses parents (imaginant par exemple qu'il est un enfant trouvé). De tels fantasmes trouvent leur fondement dans le complexe d'cEdipe $»^{2}$. Nous retiendrons ici l'aspect fantasmatique de l'élaboration de la construction, son aspect de roman, de récit ou de scénario, et son rapport à l'origine, au passé. Nous parlerons analogiquement de roman mémoriel par lequel un individu, un groupe ou une société pense son passé en le modifiant, le déplaçant, le déformant, s'inventant des souvenirs, un passé glorieux, des ancêtres, des filiations, des généalogies.

4 La mise en rapport qu'institue le syntagme « roman mémoriel » implique qu'on ait affaire à un ensemble de textes, de rites, de codes symboliques, d'images et de représentations où se mêlent dans une intrication serrée l'analyse des réalités sociales du passé, des commentaires, des jugements stéréotypés ou non, des souvenirs réels ou racontés, des souvenirs écrans ${ }^{3}$, du mythe, de l'idéologique et de l'activation d'images culturelles ou de syntagmes, vus, lus, entendus, qui viennent s'agglutiner à l'analyse. 
5 Autrement dit, pas de mémoire collective sans roman mémoriel, sans cette hybridité de formes, de syncrétisme d'un réel déjà sémiotisé, déjà pris dans l'ordre du langage et de la représentation et de l'imaginaire; pas de séparation étanche entre le scientifique et le mythe, l'explicatif et le récit, le légendaire et l'historique. C'est donc de roman mémoriel que nous allons débattre. Disons pour qu'il n'y ait pas de malentendus que le terme de roman introduit ici n'a rien de péjoratif. Il ne s'oppose pas à ce que serait un énoncé scientifique au sens positiviste du terme. Il renvoie simplement à la double structure du fonctionnement mémoriel dont nous venons de parler, structure d'hybridité, de syncrétisme, et mise en forme narrative ou symbolique, ou imagée.

Le passé, nous le savons tous, n'est pas libre. Aucune société ne le laisse livré à lui-même. La passeiité du passé est fixée. Le passé est régi, géré, conservé, expliqué, raconté, commémoré, magnifié ou haï, gardé.

Il y a d'abord un espace officiel national qui sert de cadre à cette fonction de conservation et de commémoration. Des archives, des musées, des hauts lieux comme le Panthéon, toutes sortes de collines inspirées où souffle l'esprit national, des rues au nom symbolique magnifiant des héros, des événements, des lieux, des dates; des cimetières et des cimetières militaires en particulier, des plaques, des monuments de la victoire, aux morts, aux grands hommes. Il s'agit très précisément des «lieux de mémoire " selon la belle expression de P. Nora et de son équipe ${ }^{4}$. La mémoire nationale ou officielle est encore balisée par une temporalité propre. Elle est scandée par ses dates, ses jours fériés, ses fêtes et en particulier ses fêtes nationales qui sont le rappel des temps héroïques et qui constituent un temps épique, un retour aux origines, au légendaire national.

Le passé régi est enfin pris dans un ensemble de gestes, d'images et de rites qui marquent le corps : rites des inaugurations, pose des plaques, des premières pierres, coupures de rubans associés avec des hymnes nationaux exigeant une posture du corps, debout, recueilli et digne; hommages; minutes de silence et commémorations multiples. Tout cela dessine un milieu d'évidences façonnées, contribue à la création d'un «habitus national » fait de récits et de rites. Il s'agit d'une gestion des traces ${ }^{5}$, une gestion des « cendres de conséquences » ou anonymes, d'une gestion de la saga identitaire, saga de la continuité de la nation et de l'État et de sa légitimité. Gestion sur le mode épique, public, monumental ${ }^{6}$.

9 En face de ou à côté de, ou contre la mémoire nationale (cela dépend des pays, des régimes et des conjonctures), la mémoire savante construite par les historiens qui est plus un travail d'élaboration des traces qu'une gestion de ces traces. Il ne s'agit pas ici de reprendre de vieux débats sur l'histoire ou de récents ouvrages de synthèse qui, avec des points de vue différents, ont renouvelé notre façon de poser les problèmes. Je voudrais évoquer ici simplement pour mémoire Michel de Certeau ${ }^{7}$, P. Ricœur ${ }^{8}$ et Hayden White ${ }^{9}$.

L'histoire raconte, explique, elle est la science de l'intelligibilité du passé. Elle met en intrigues des actions, des phénomènes complexes, des rapports. L'histoire comme discipline institutionnalisée est plongée dans des conjonctures historiques mouvantes; formée, informée, déformée par la mémoire nationale (mais elle agit également sur elle en retour) ; à l'écoute des mémoires collectives qu'elle renie, nie ou dénie ou au contraire qu'elle contribue à mettre sur la place publique, qu'elle prend en charge, auxquelles elle donne une nouvelle légitimité.

11 Il s'agit, comme l'exprime très bien Roger Chartier, de 
«discours dépendant d'un ensemble de procédures techniques, de règles de validation, de disciplines de contrôle qui, pour varier historiquement, n'en sont pas pour autant arbitraires ou subjectives, et d'autre part [d']un savoir traversé par les contraintes qui lui viennent du lieu social où il s'élabore et les obligations de son écriture propre ${ }^{10}$."

Enfin, la mémoire collective, le légendaire de groupe, la mémoire vivante du vécu comme le disait Halbwachs :

«Qu'est-il arrivé en France et dans le monde en 1877, l'année de ma naissance? C'était l'année du 16 mai quand la situation politique instable donna naissance vraiment à la III République. De Broglie était au pouvoir et Gambetta déclara "Il faudra se soumettre ou se démettre". Le peintre Courbet mourut. Victor Hugo publia son second volume de La Légende des siècles. Le boulevard Saint-Germain fut achevé à Paris tandis qu'on commença la construction de l'avenue de la République. L'attention de l'Europe se concentra sur la guerre russo-turque. Osman Pasha fut contraint de lâcher Plevna après une longue et héroïque défense. Faisant cela, je reconstitue un cadre spatial dans lequel je me sens complètement perdu ${ }^{11}$."

13 C'est que la mémoire collective n'est ni chronologique, ni distanciée. Elle peut être informée par le savoir historien, dominée par la mémoire nationale, mais elle a son domaine spécifique, le légendaire du groupe, une mémoire à la fois tenace et floue. Elle aussi conserve, garde, commémore les traces, sa temporalité est cyclique et/ou uchronique, symbolique, mêlant les lieux et les dates, les confondant. La mémoire collective juxtapose l'acuité du détail dans la quotidienneté et le trou de mémoire sur l'événement précis. Uchronique avons-nous dit car, de souvenirs-écrans en souvenirsécrans, elle déplace les décors, les costumes, les événements. À propos d'une enquête sur la Seconde Guerre mondiale, Dominique Veillon rapporte le phénomène suivant: une femme au cours d'une des séances de son récit de vie évoque l'entrée des troupes allemandes dans une petite bourgade où elle se trouvait, en parlant des "casques à pointe » qu'elle voyait défiler. L'auteur de l'article fait le commentaire suivant :

«Manifestement dans cette description il y a confusion, substitution d'une guerre à l'autre pour ne laisser subsister que ce qui préexistait en elle [l'interviewée], en l'occurrence ce que sa mère lui avait dit, ce qu'elle avait appris de ses grandsparents. La suite du récit prouve assez bien, avec le rappel des pillages et des exactions commises en 1870 et 1914 par les Allemands qu'elle appelle les "Germains"12."

$\mathrm{Au}$ contraire de l'historicité chronologique, la mémoire collective fonctionne à la «madeleine» de Proust, par associations ou par mobilisation d'un sens déjà-là. Seul compte en effet le sens à donner au passé. De là ce goût pour les symboles, les allégories de la mémoire collective, gardienne des valeurs, de la tradition, et de l'interprétation du passé du groupe. Si son espace-temps est local, symbolique, cyclique et uchronique, elle se fait volontiers élégie, péan ou panégyrique pour représenter sa propre épopée, à moins qu'elle ne se donne dans la dispersion désordonnée d'associations d'idées ayant comme point d'appui le vécu. Il faudrait alors distinguer, pour y voir plus clair, une mémoire collective du groupe minoritaire élaborant son roman mémoriel pour mieux conforter son identité menacée ${ }^{13}$, et un sens plus vague et plus englobant de la mémoire collective que j'appellerais mémoire culturelle, qui se donnerait à l'individu sous la forme de « Je me souviens » de G. Perec, ou de cette remarque de Stendhal :

«En écrivant ma vie en 1835, j’y fais bien des découvertes. Ces découvertes sont de deux espèces. 1) D'abord, ce sont de grands morceaux de fresques sur un mur, qui depuis longtemps oubliés apparaissent tout à coup, et à côté de ces morceaux bien conservés, sont comme je l'ai dit plusieurs grands espaces où l'on ne voit que la 
brique sur le mur. L'éparvérage, le crépi sur lequel la fresque était peinte est tombé, et la fresque est à jamais perdue. À côté de ces morceaux de fresque conservés, il n'y a pas de date, il faut que j'aille à la chasse des dates, actuellement en 1835 . Heureusement, peu importe un anachronisme, une confusion d'une ou deux années. À partir de mon arrivée à Paris en 1799, comme ma vie est mêlée avec les événements de la gazette, toutes les dates sont sûres. 2) En 1835, je découvre la physionomie et le pourquoi des événements ${ }^{14}$.»

L'individu dans tout cela, pétri par les images-forces de la mémoire nationale d'un côté, par le récit familial, la saga des parents et grands-parents, par ses généalogies plus ou moins imaginaires, par les albums de photos, menus objets conservés, les bribes de correspondance d'autre part, informé par le savoir des historiens même vulgarisé, par cette forme de savoir que diffusent les média, le cinéma, la littérature légitime ou arlequinisée, l'individu marqué par tous ces scénarios qui peuvent se renforcer l'un l'autre mais aussi se contredire, l'individu dis-je, bricole comme il peut sa représentation du passé, son imagerie, son récit, dans l'ordre d'un moule narratif obligé ou dans la dispersion de souvenirs-flash, dans un sens préétabli dans un combat identitaire, dans une contre-mémoire fragmentaire, ou dans une dispersion de mémoires migrantes.

Récapitulons quelque peu, dans le jeu de cette élaboration d'un roman mémoriel, les types de mémoire que nous avons dégagés : une mémoire nationale (M1), une mémoire savante (M2), une mémoire collective (M3), une mémoire culturelle (M4).

Première constatation : il n'y a pas de cloison étanche entre ces types de mémoire, au contraire. Nous avons affaire à une grande circulation discursive et mémorielle des formes d'appropriation du passé, à des rapports de hiérarchisation, selon les moments, les régimes et les conjonctures. Toutes ces mémoires contribuent à leur façon à l'écriture du roman mémoriel, à ce tissu composite fait d'images, de phrases, de syntagmes, d'extraits de manuels scolaires, de films, de chansons, d'idées qu'une société se fait de son propre passé.

Seconde constatation: chaque type de mémoire relève d'un chronotope particulier au sens que M. Bakhtine donne à ce terme, c'est-à-dire d'une condensation spécifique de l'espace-temps qui constitue le type de mémoire considéré en genre spécifique.

La mémoire nationale relève d'un espace-temps monumental, public et épique. Il s'agit de la geste du temps origine des héros nationaux, d'une grande saga de fondation. La mémoire collective de groupe, plus privée et plus locale, partage avec la mémoire nationale le caractère épique de son chronotope, une temporalité cyclique, uchronique, mythique. Ces deux mémoires se rapportent à ce que M. Bakhtine appelle l'épopée ou la parole des pères :

"L'épopée, genre précis, comporte trois traits constitutifs : 1) Elle cherche son objet dans le passé épique national, le "passé absolu" selon la terminologie de Goethe et de Schiller. 2) La source de l'épopée c'est la légende nationale (et non une expérience individuelle et la libre invention qui en découle). 3) Le monde épique est coupé par la distance épique absolue du temps présent : celui de l'aède, de l'auteur et de ses auditeurs ${ }^{15}$.»

20 À l'opposé, le savoir historien (la mémoire M2) développe un espace multi-dimensionnel et une temporalité chronologique et/ou périodisée dans un effet de distanciation entre le passé et le présent. Il s'agit du chronotope d'un discours explicatif, métadiscursif, argumentatif, rationnel. Reste la mémoire culturelle M4, potentiellement polyphonique qu'elle se donne dans le flash du souvenir, dans l'ordre narratif chronologique ou dans le narratif métaphorique, mais qui a besoin de figuralisation. Elle n'est que potentielle. Sa 
mise en figure, sa mise en texte, je la vois infiniment plus développée dans le cadre de la fiction que dans le cadre du dispositif qui débouche sur le récit de vie ${ }^{16}$.

Passé figé, fixé, conservé, magnifié, commémoré. Le passé pèse. Il y a une pesanteur de la mémoire, un encombrement de l'esprit. Nietzsche, dans les Considérations intempestives, écrivait :

«L'Histoire est le bien de l'homme qui veut conserver et vénérer le passé, de celui qui jette un regard un regard frileux et aimant vers les origines, vers le monde où il a grandi. Par cette piété, il s'acquitte en quelque sorte de sa dette de reconnaissance envers le passé. Mais attention à l'excès d'Histoire, attention à une piété trop grande. Si l'homme se livre à cette curiosité nostalgique et quasiment religieuse et sombre ainsi dans le culte des morts, alors il étouffe, le passé l'alourdit. Cette pesanteur du passé l'empêche justement de créer. Il a besoin d'une légèreté, d'une innocence, d'une sorte d'insouciance pour créer ${ }^{17}$. »

Il peut en effet y avoir captation mortifère du passé et à ce moment-là, le passé sur le plan fantasmatique doit se transformer pour diverses raisons, pour combler l'abîme hallucinatoire d'une origine perdue, pour combler un manque, ou pour s'adapter à une conjoncture qui réclame un autre passé. Le roman mémoriel se transforme.

\section{Le passé réaménagé}

Les modalités à travers lesquelles la trace bouge, se remodèle, sont multiples. J'en présenterai ici quelques-unes parmi les plus connues.

D'abord le silence, le silence structuré si bien étudié par L. Passerini ${ }^{18}$. Le silence refus, le silence tabou, le silence sur ce qui gêne et sur ce qui fait mal. La chose nommée prend corps. Non verbalisée, elle reste dans le flou. Tous ceux qui ont travaillé en histoire orale ont rencontré ces moments où la personne déclare « je ne parlerai pas de ça », volontaire, décidée, ou au contraire ces moments où elle voudrait parler mais sa voix s'étrangle et c'est le silence qui s'installe. Moments d'émotion intense voire intolérable.

Le silence qui permet le refoulement, le déni ou l'oubli. La psychanalyse nous a appris que c'était à ces moments-là que quelque chose d'important se passait, qu'une résistance énorme était rencontrée. Le silence et son cortège d'associés, l'oubli par refoulement et qui par conséquent peut être levé, l'oubli libérateur ou aliénant, enfin la négation du passé par dénégation, refus de savoir, négation volontaire comme la remise en question de l'existence des chambres à gaz et de la tragédie du Génocide.

Mieux connues, mieux étudiées, les réécritures politiques de l'histoire comme dans l'URSS stalinienne et post-stalinienne, la réécriture du rapport entre le peuple russe et les peuples non russes après la liquidation de l'école historique de Prokovskii dans les années 1930, les réécritures périodiques de l'histoire du Parti, la disparition de certains personnages des photographies officielles ${ }^{19}$, Trotskii, Boukharine, etc., l'élimination des dictionnaires et encyclopédies du nom des victimes des purges de 1937, etc.

Réécriture, mais aussi relecture du passé en fonction d'une utilisation politique immédiate, ou en fonction d'élaborations idéologiques plus complexes. Deux exemples rapides. En 1980, la Province du Québec soumet ses habitants à un referendum sur le thème de la souveraineté-association avec le Canada, ouvrant par là une voie possible à l'indépendance du Québec à moyen terme. Le referendum est précédé d'un long débat, d'une campagne électorale peu commune où l'histoire, le passé joue un rôle de premier plan. On se bat à coups de passé. Sur les murs de Montréal, cette affiche tout droit sortie 
d'un ancien manuel scolaire où l'on voit Madeleine de Verchères, héroïne du XVII siècle ayant courageusement arrêté la progression des Indiens, et cette légende : « Madeleine de Verchères aurait-elle dit OUI ?»

L'autre exemple a trait à l'idéologie, la mythologie politique de Begin et dont il a usé et abusé jusqu'à son départ du pouvoir ${ }^{20}$. À partir du passé tragique du peuple juif et en particulier du Génocide de la dernière guerre mondiale, Begin se forge tout un discours qui a sa cohérence interne accrochée à l'interprétation du passé. Il s'agit, comme le soustitre du livre auquel je fais allusion l'indique, d'un mythe de la rédemption par le retour, par l'établissement d'un État et d'un État exclusivement juif. L'auteur de l'ouvrage défend la thèse selon laquelle la réponse ou les réponses à l'horreur de l'Holocauste ne pouvaient qu'être mythiques voire théologiques. Le premier volume des mémoires de M. Begin, La révolte ${ }^{21}$, est construit comme les aventures d'un héros selon la formule : séparation de l'objet de valeur, initiation et retour. Toute la pensée politique obsessionnelle va dépendre de cette représentation d'une odyssée tragique, depuis l'Holocauste jusqu'au retour à Sion.

Autre relecture de l'histoire, non pas directement politique celle-là mais venue de l'institution historienne, celle de la réévaluation du passé nazi en Allemagne. Ernst Nolte est un historien connu' ${ }^{22}$. Quant à M. Andreas Hillgruber, il a beaucoup écrit sur la stratégie militaire hitlérienne. Récemment, les deux auteurs se sont lancés dans une réévaluation du régime nazi qui surprend. Sous le prétexte cohérent et à mes yeux fort juste pour un historien de traiter la société nazie avec objectivité, comme une autre, de l'étudier dans toutes ses dimensions, y compris sa quotidienneté, sous ce prétexte, s'instaure une entreprise de banalisation du régime, banalisation d'autant plus grande qu'on fait ressortir que le véritable ennemi était le bolchevisme, que le goulag avait innové en matière d'extermination et avait le privilège de l'antériorité. On ne se contente plus, comme il y a vingt ans, de renvoyer dos à dos l'URSS stalinienne et l'Allemagne hitlérienne dans une même horreur totalitaire, désormais l'Allemagne apparaît plus «normale » ou, comme le dit un journaliste, on peut « refouler Hitler grâce à Staline et Pol Pot $»^{23}$.

Autre réévaluation entraînant des bougés de l'interprétation du passé en fonction de larges conjonctures politiques et culturelles : la levée de censure ces dernières années en France qui permet de parler de la collaboration à la limite de la réhabilitation : passage de M. Bardèche à l'émission « Apostrophes » où, en toute impénitence, il tient les propos qu'il tient sur l'histoire en renvoyant tout le monde dos à dos, biographie innommable d'Anne Brassié sur R. Brasillach (à quand en "10/18" ou en "Folio" Gallimard Les décombres de Rebatet ${ }^{24}$ ?), argumentations du défenseur de Barbie, cherchant lui aussi à banaliser le Génocide des Juifs pendant la dernière guerre et à nous présenter Barbie comme un vieil homme malade qu'on importune inutilement.

31 Dernier exemple de ce type de réinterprétation du passé en fonction de conjonctures politiques nouvelles, la réaction d'une partie de la population soviétique en face non seulement des réformes de Gorbatchev, mais du nouvel état d'esprit qui entoure ces réformes. On peut y voir dans le domaine culturel du moins une lutte pour retrouver une vraie mémoire, un vrai passé.

32 La Literaturnaia Gazeta formulait le 22 octobre 1986 le programme suivant : " Si nous nous tournons vers le passé, c'est pour pouvoir recevoir des réponses aux questions qui nous torturent». Parmi ces questions brûlantes, le stalinisme, Staline, non seulement 
l'héritage de la planification centralisée mais les purges, l'arbitraire, la collectivisation, et un état d'esprit qui freine l'esprit et la mise en pratique des réformes. Cette interrogation sur le stalinisme est un des éléments-clé du débat d'aujourd'hui. En témoigne ce roman d'Anatoli Rybakov, Les enfants de l'Arbat, que la revue Druzhba Narodov a publié et qui paraîtra sous peu en format de livre. Le roman a attendu vingt ans pour voir le jour. La très libérale Novyi Mir dirigée à l'époque par A. Tvardovskii devait le publier en 1967, puis Oktiabr' devait à son tour le publier en $1979 . .$.

Le héros du roman est Sasha Pankratov, un dirigeant du Komsomol, qui se fait arrêter un beau jour de 1934, sans doute sur dénonciation calomniatrice dirait Joseph K. Il est interrogé par la police secrète, le NKVD, et condamné à un exil sibérien. Quelques-uns de ses amis tentent d'organiser une pétition en signe de protestation. Fort peu la signent : la plupart trouvent les prétextes les plus divers pour ne pas signer. Parallèlement à cette intrigue principale qui met en scène l'univers des délateurs, agents d'information, tribunaux, interrogatoires, etc., l'auteur nous présente une seconde scène narrative qui a pour personnage Staline, son monologue intérieur, sa paranoïa, son désir d'ordre et de pouvoir. Le livre se termine sur l'assassinat de Kirov en décembre 1934, signal d'une nouvelle période d'arrestations arbitraires.

On s'est arraché à Moscou les numéros de la revue et l'on attend avec impatience le livre, et en Occident sa traduction ${ }^{25}$.

L'interrogation sur le stalinisme est partout. Boris Mozhaev dans Muzhiki $i$ baby (Les moujiks et leurs femmes, 1988), met en scène la collectivisation de la fin des années 1920 et le coût humain et social de celle-ci. «La disparition », nouvelle inachevée de I. Trifonov (Druzhba Narodov, 1, 1987), présente les garçons de La maison sur le quai, en 1937. Elle est réservée aux membres du Parti et à leurs familles. Dans les intérieurs, partout des tapis de Perse, et à l'extérieur, de magnifiques voitures de luxe pour les dirigeants. Et le peuple dans tout ça ? À l'écran c'est Repentir de T. Abuladze, et bien d'autres.

Cette remise en question du stalinisme s'accompagne d'un retour à Lénine sans mythification, d'un Lénine sans mausolée si l'on veut. Pour le $70^{\mathrm{e}}$ anniversaire de la Révolution d'Octobre, Gorbatchev proposait qu'on mît fin aux «pages blanches » et à l'omission de certains noms dans les histoires officielles et dans les commémorations. Dans Tak pobedim! Shest pes o Lenine (Retouches au portrait de Lénine, 1985) de M. Chatrov, on voit Martov, Kollontaï, Zinoviev, Boukharine et Trotski, ce qui n'est pas banal et marque la volonté de s'approprier un passé vrai.

En face, il n'y a pas seulement les attentistes qui ne veulent pas parler ou restent prudents, ne sachant pas combien de temps le gorbachévisme va durer, il n'y a pas seulement les vieux de la vieille, vieux militants staliniens ayant joué un rôle peu avouable dans l'État, l'administration, l'armée ou la police, il y a aussi une partie de la population qui participe (et pas seulement en Géorgie) du mythe de Staline. Dans une période de grandes incertitudes, de grandes difficultés, au sein de relations internationales tendues, Staline représente une valeur refuge, refuge du nationalisme, du sentiment de grande puissance, refuge de ceux qui aiment l'ordre et puis quelque chose de plus vague, chez les jeunes en révolte une figure identificatoire contre toutes les valeurs actuelles. Dans le dernier roman de T. Aïtmatov (Plakha), un jeune illuminé, Abdias, est jugé, mis à mort par les membres d'une bande de trafiquants de drogue, recrutés pour chasser des antilopes. Ces trafiquants se servent de Staline comme valeur modèle et comme aune à laquelle rapporter leurs jugements : 
« Nous on suit fidèlement les directives gouvernementales, et toi cafard, tu veux saboter le plan! Tu es un ennemi du peuple, voilà ce que tu es! Un ennemi du peuple et de l'État. Et les traîtres comme toi, il n'y a pas de place pour eux sur cette terre. "Celui qui n'est pas avec nous est contre nous", c'est Staline qui l'a dit. Ce genre de racaille il faut l'exterminer sans pitié ! [...] Tu crois peut-être que si Staline est mort, on doit plus toucher aux mecs de ton espèce ${ }^{26}$ ?" en sciences humaines, les journaux intimes, l'autobiographie sous toutes ses formes, les récits de vie, les éléments empruntés aux approches biographiques en histoire, en sociologie ${ }^{27}$, en ethnologie, la biographie en tant que telle enfin. Il s'agit de genres très à la mode, encombrant les tables et les étagères des librairies, et dont il faut bien saisir qu'ils s'inscrivent dans un énorme retournement de la conjoncture intellectuelle et culturelle. Écroulement des grandes idéologies, dit-on peut-être un peu vite, mais à tout le moins écroulement des systèmes explicatifs globaux, refoulement de la totalité considérée comme totalitaire et de la causalité devenue dogme, enfermement. À la limite, refus de la rationalité comme valeur. D'où un certain nombre de déplacements des objets en histoire: plus personne ne s'intéresse aux rapports sociaux, aux trends économiques, chacun veut écrire une histoire de la sensibilité, du goût, des odeurs, des couleurs, des valeurs; déplacement des méthodes: on délaisse le quantitatif, le sériel, le statistique pour promouvoir le qualitatif, la singularité; déplacement enfin des principes d'explication: le phénoménologique et l'herméneutique prenant peu à peu la place des types d'explication traditionnels. Cela donne aussi dans le grand public un goût du localisme, de l'anecdotisme, du recentrage sur l'individu au nom de cette «ère du vide " si bien mise en valeur par Gilles Lipovetsky ${ }^{28}$. Depuis quelques années, on nous annonce que la littérature et plus particulièrement le roman sont menacés, en grand danger, que peut-être devant la médiatisation générale il faut capituler. La première de cette menace est due à la mort de l'avant-garde, à l'exténuation de toutes les expérimentations, à un appauvrissement de l'écriture du signifiant, aux fatigues du formalisme, du modernisme ou du postmodernisme. Une littérature qui finirait par se prendre comme objet, qui jouerait perpétuellement de la parodie, du second degré, de la mise en abyme, une littérature qui aurait banni l'intrigue, les personnages, le récit, pour ne s'attacher qu'à l'écriture, aux structures d'agencements, aux rythmes, bref, tout ce qui faisait depuis les années 1920, dans le bruit et la fureur, la 
légitimité des cercles restreints, est en train de s'exténuer, de s'isoler, de s'étioler. Dans son discours de Stockholm, lors de la remise du prix Nobel, Claude Simon déclare :

«Lorsque je me trouve devant ma page blanche, je suis confronté à deux choses: d'une part le trouble magma d'émotions, de souvenirs, d'images qui se trouvent en moi, d'autre part la langue, les mots que je vais chercher pour le dire, la syntaxe par laquelle ils vont être ordonnés et au sein de laquelle ils vont en quelque sorte se cristalliser. Et tout de suite, un premier constat : c'est que l'on n'écrit (ou ne décrit) jamais quelque chose qui s'est passé avant le travail d'écriture, mais bien ce qui se produit (et cela dans tous les sens du terme) au cours de ce travail, au PRESENT de celui-ci, et résulte, non pas du conflit entre le très vague projet initial et la langue, mais au contraire d'une symbiose entre les deux ${ }^{29}$. "

Mais la plupart des critiques français sont restés stupéfaits à l'annonce de l'attribution du prix Nobel de littérature à Claude Simon. Pour eux c'est la mort du roman qu'on couronnait.

Le second danger qu'on dénonce, aussi bien en peinture qu'en littérature, serait le retour au naturalisme, aux plates conventions du XIX ${ }^{e}$ siècle, aux Bouguereau, à l'art pompier et autres «porteuses de pain» qui seraient devenus à la mode, doubles scripturaires et picturaux de l'échec de l'architecture des Halles, du quartier de l'Horloge ou de tous les espaces piétonniers à Paris. Le grand danger démagogique serait celui du kitsch défini par Milan Kundera dans les termes suivants :

«Le mot kitsch désigne l'attitude de celui qui veut plaire à tout prix et au plus grand nombre. Pour plaire, il faut confirmer ce que tout le monde veut entendre, être au service des idées reçues. Le kitsch, c'est la traduction de la bêtise des idées reçues dans le langage de la beauté et de l'émotion. Il nous arrache des larmes d'attendrissement sur nous-mêmes, sur les banalités que nous pensons et sentons. Après cinquante ans, aujourd'hui, la phrase de Broch devient encore plus vraie. Vu la nécessité impérative de plaire et de gagner ainsi l'attention du plus grand nombre, l'esthétique des mass media est inévitablement celle du kitsch; et au fur et à mesure que les mass media embrassent et infiltrent toute notre vie, le kitsch devient notre esthétique et notre morale quotidiennes ${ }^{30}$. »

Pour sortir de ces deux alternatives néfastes, là encore depuis quelques années, la critique s'est tournée vers Vienne, vers l'Europe centrale, vers un art du roman venu d'une tout autre tradition. Musil et Broch étaient les deux grandes figures tutélaires parce que leur œuvre se tenait à égale distance des faderies naturalistes et de l'expérimentation formaliste. À la fois œuvre d'idées, d'échos philosophiques, œuvre romanesque au sens plein du terme, elles se laissaient traverser par de multiples voix, idéologies, rythmes et discours. Cette polyphonie généralisée, cette hybridité très proche de Bakhtine, ce cosmopolitisme intégrant tous les exils comme seule position d'écriture, semblaient le seul recours qui permît de mettre fin aux idéologies de l'enracinement, et qui ouvrît l'espace du roman au transverse, à un espace multiculturel, pluriel, à une respiration libre. La voie viennoise ou la représentation imaginaire de ce qu'avait pu être Vienne, c'était aussi une façon de prendre acte dans l'esthétique de la prétendue mort des idéologies. Comme le dit très bien Guy Scarpetta dans L'impureté :

"S'affranchir des idéologies modernistes ou avant-gardistes, c'est aussi s'émanciper des grands systèmes utopiques, issus du xIxe siècle, auxquels elles furent le plus souvent liées [...] Autrement dit, il me semble que ce qui "revient" aujourd'hui dans tout un pan de l'art et de la littérature, c'est l'affirmation d'une impureté fondamentale - pas très éloignée, peut-être, de ce que les discours religieux désignaient comme le péché originel. Façon de renouer, sans doute, avec cette fonction de l'art que l'avant-gardisme avait plus ou moins refoulée: dire le 
Mal, désigner le négatif du lien social, l'envers des optimismes idéologiques et des utopies communautaires ${ }^{31}$." maisons d'édition, qui sont parfois primés, parfois pas, mais toujours accueillis par la critique qui leur consacre de nombreuses pages dans les principaux quotidiens et dans les magazines littéraires. Ces romans viennent des horizons les plus divers. Certains de leurs auteurs sont de grands noms de l'institution littéraire, d'autres sont moins connus. Je veux parler, pêle-mêle, de Le miroir qui revient (Minuit) d'Alain Robbe-Grillet, de L'amant (Minuit) de Marguerite Duras, du Valet de nuit (Grasset) de Michel Host, de La vie fantôme (POL) de Danièle Sallenave, de Le salon du Wurtemberg (Gallimard) de Pascal Quignard, de Prise d'otage (Denoël) de Marcelin Pleynet, et de Course d'amour pendant le deuil (Gallimard) de Florence Delay. Cette liste bien entendu, n'a rien d'exhaustif. Elle signale un problème, elle balise un symptôme littéraire et sociologique. Plusieurs de ces écrivains viennent du cercle restreint de l'avant-garde ou de l'expérimentation. Ils ont tous fait le même chemin de façon consciente ou inconsciente. Ils veulent être lus par le grand public et pour cela ils réinscrivent une intrigue, un récit, des personnages et ont recours à une écriture qui rappelle (tout en prenant pour acquise la période formaliste) le code réaliste, voire naturaliste. Dans sa définition du kitsch, Milan Kundera poursuivait :

"Jusqu'à une époque récente, le modernisme signifiait une révolte non conformiste contre les idées reçues et le kitsch. Aujourd'hui, la modernité se confond avec l'immense vitalité mass-médiatique, et être moderne signifie un effort effréné pour être à jour, être conforme, être encore plus conforme que les plus conformes. La modernité a revêtu la robe du kitsch ${ }^{32}$. »

Le retour du lisible aujourd'hui serait en somme cette rencontre de la modernité et du conforme, du plaire-à-tout-prix. Dans Le miroir qui revient, Robbe-Grillet nous raconte la chronique familiale de son horrible famille fasciste dans une structure somme toute linéaire et sans mystère, tout en truffant son livre d'intrusions d'auteur sur l'écriture et le pourquoi de ses transformations d'écriture. L'autobiographie est impossible, bien entendu, mais on n'échappe pas à la vague du biographique, du narcissisme, de ces "tyrannies de l'intimité » dont on sait depuis longtemps à quel point elles sont pernicieuses. Le miroir qui revient pourrait bien être aussi celui de Stendhal et de l'esthétique réaliste. Quel paradoxe de la part d'un écrivain qui a commencé sa carrière en désignant à tous Balzac comme l'ennemi à abattre. Marcelin Pleynet est associé à l'entreprise moderniste, à l'expérimentation, à Tel Quel. Prise d'otage est un roman qui distribue des thèmes à la mode, le terrorisme en Italie, et le "light porn», expression intraduisible. Confusion des genres, des écritures, le roman réinscrit bien le souvenir de l'écriture moderniste avec ses longues phrases, ses récurrences, ses points de suspension, mais c'est pour mieux nous piéger dans son affaire d'otage.

Danièle Sallenave, qui elle aussi vient d'un horizon d'expérimentation, nous présente dans La vie fantôme une histoire d'adultère banale et sans relief, quelque chose de notre médiocrité d'aujourd'hui, disait un critique, notre monde petit-bourgeois sans horizon. La couverture du livre montre un lit aux draps froissés, sur la moquette des souliers de femme. Il est bien connu que dans les époques de crise, sans horizon, sans idéal, sans dessein sociétal, il ne reste que le lit. Le roman en est l'expression la plus achevée, dans une écriture lisse, sans aspérités. Pascal Quignard vient également d'un cercle restreint et de l'expérimentation. Le salon du Wurtemberg se lit avec plaisir. On y rencontre les couleurs pastel des comptines et des bonbons au nom magique. Le travail du souvenir y est remarquable. Mais là encore, l'intrigue marquée par des dates précises, des repères, 
finit par tout emporter. L'intrigue n'est plus le support de l'écriture mais devient la définition du romanesque ${ }^{33}$.

La littérature participe donc de ce grand remaniement qui ramène au lisible. Pourtant, je le répète, même dans ce grand mouvement régressif, la fiction bat en brèche l'illusion biographique, la continuité et l'homogénéité. Elle seule jusqu'à présent sait «laisser battre le discontinu " selon la belle expression de D. Oster ${ }^{34}$. Même le nouveau RobbeGrillet se sent obligé d'ironiser sur le roman biographique qu'il se propose à écrire :

"Quand je relis des phrases du genre "ma mère veillait sur mon difficile sommeil" ou "son regard dérangeait mes plaisirs solitaires", je suis pris d'une grande envie de rire, comme si j'étais en train de falsifier mon existence passée dans le but d'en faire un objet bien sage, conforme aux canons du regretté Figaro littéraire: logique, ému, plastifié. Ce n'est pas que ces détails soient inexacts (au contraire peut-être). Mais je leur reproche à la fois leur trop petit nombre et leur modèle romanesque, en un mot ce que j'appellerai leur arrogance. Non seulement je ne les ai vécus ni à l'imparfait ni sous une telle appréhension adjective, mais en outre, au moment de leur actualité, ils grouillaient au milieu d'une infinité d'autres détails, dont les fils entrecroisés formaient un tissu vivant. Tandis qu'ici j'en retrouve une maigre douzaine, isolés chacun sur un piédestal, coulés dans le bronze d'une narration quasi historique (le passé défini lui-même n'est pas loin) et organisés suivant un système de relations causales, conforme justement à la pesanteur idéologique contre quoi toute mon œuvre s'insurge ${ }^{35}$. $"$

Et la place des récits de vie dans ce grand paradigme du biographique? Elle peut être centrale, car en lui s'articulent le récit individuel, les habitus, les moules narratifs, les divers stéréotypes et les différents types de mémoire que j'ai évoqués ${ }^{36}$. Le récit de vie révélera les trous, les oublis, les silences, les interactions entre le vécu, le déjà-connu, le raconté et le transmis, il mettra en avant l'encadrement dont il est l'objet et les différents modèles identificatoires qui le constituent. Il est décisif comme maillon de la construction du roman mémoriel. Je proposerais simplement qu'on ne l'étudie pas seul, comme fin en soi, si passionnant qu'il puisse être. Je voudrais qu'on le rapporte à cette circulation discursive et mémorielle qui balise à la fois la mémoire nationale, la mémoire savante, la mémoire collective et la mémoire culturelle. En intégrant le récit de vie à cette circulation discursive, quatre questions pourraient lui être adressées. Y a-t-il conformité, synchronie entre le récit de vie et ce que l'on sait par ailleurs des différents types de mémoire? De laquelle le récit de vie se rapproche-t-il le plus, ou encore, comment ces différentes mémoires le traversent-elles et dans quelle mesure? Y a-t-il ambivalence, symptôme d'une résistance? Luisa Passerini rencontre cette ambivalence chez les ouvriers de l'usine Fiat-Miraflori à Turin. Elle met en relief
« le cadre d'un conflit au plan symbolique, entre le régime et les cultures populaires des classes laborieuses. Les attitudes verbales et les comportements ainsi reconstruits (comme le port de la cravate rouge ou de la chemise noire, le chant de chansons socialistes ou d'hymnes fascistes dans une version ironique, les graffiti et les jurons) montrent à la fois une résistance et une compensation symbolique de l'acceptation. L'ambivalence est maximale dans les manifestations propres du rire qui vont du bon mot contre le régime jusqu'à l'auto-dénigrement et à la reprise de formes du répertoire comique populaire ${ }^{37}$.»

Seconde question : quel est le chronotope, l'espace-temps particulier du récit de vie ou de tel récit de vie? Est-il chronologique, est-il uchronique, mythique-cyclique ? Balaie-t-il toutes ces formes? Le trouve-t-on à des moments différents du récit, mais alors pourquoi passe-t-on de l'uchronie à la chronologie et de la chronologie au cyclique? Quel rapport peut-on établir entre la temporalité inscrite et le contenu? Troisième question touchant 
à l'épaisseur narrative du récit. Est-il monologique ou au contraire polyphonique? Arrive-t-il à se construire un espace lui permettant d'inscrire plusieurs voix, plusieurs modèles identitaires, les différentes mémoires qui le constituent ? Ou n'y arrive-t-il pas ${ }^{38}$ ? Dernière question enfin, liée à la précédente : quelle fonction le récit de vie privilégie-til dans le cadre de la circulation discursive liée au passé : fonction socio-idéologique, médiatique, fantasmatique, thérapeutique?

51 Il me semble que cela nous permettrait d'avancer. Sans oublier jamais de briser avec l'illusion biographique, avec les pièges de la cohérence et de la continuité, et en ayant présent à l'esprit que le trou de mémoire, si essentiel pour nous, n'a pas pu être comblé. Je voudrais évoquer pour finir ce très beau texte de Serge Doubrovsky qui a précisément pour titre "Trou de mémoire " ${ }^{39}$. Le narrateur seul à Paris regarde aux «Dossiers de l'écran " une émission sur le Journal d'Anne Frank. À cette occasion, toutes sortes de souvenirs et de pensées le submergent. Il décide d'aller à l'Étoile le lendemain, 8 mai 1985, pour fêter la victoire. Il évoque déjà ce que sera la cérémonie. Le lendemain, il s'affale sur son divan et regarde la cérémonie en direct sur TF1, bien qu'il habite à un quart d'heure seulement de l'Étoile. À la fin de la retransmission il décide d'y aller enfin, à l'Étoile. Il s'approche de la flamme, déambule, toujours assailli par des souvenirs, par le cours de sa vie présente et la pensée de son vieillissement. Puis soudain cette question : « Le 8 mai 45, j'étais où? J'ai fait quoi?». On excusera la longueur de la citation, il s'agit d'un texte impossible à couper et qui rend parfaitement compte de ce que j'appelle le roman mémoriel :

«En moi que du silence. Du noir. Sur le petit écran portatif dans ma caboche, pas une image n'apparaît. Au lieu du spectacle son et lumière, rien. Ma projection intérieure est en panne. J'y crois à peine. Pas Dieu possible. Ce jour de Dieu, TE DEUM des athées. Liesse délirante, messe universelle. La kermesse, j'ai dû faire une de ces foires. Ça foire. Pas mèche. Pas une ombre ne s'agite. Saisi d'angoisse, je tâtonne avec une hâte fébrile. Je m'ausculte, je palpe la mémoire. Je bats le rappel des souvenirs. Je retrouve illico l'enveloppe jaunie, qualité papier journal, de l'époque. Dedans une autre enveloppe, toute petite. Dans cette enveloppe, une simple carte. Sur cette carte, une simple signature. CHARLES DE GAULLE. Pas plus. Suffit. Vous ne pouvez pas vous rendre compte. Je sors de mon trou à rat. Une larve, une vermine, un Juif. Pas même un sous-homme, une sous-merde. Je tiens à peine sur mes jambes. Entre les jambes, des BK aux couilles qui me lancinent nuit et jour. De sa propre main, en 45, il a pris la peine, Charles de Gaulle. J'en explose encore. Concours général, le premier de l'après-guerre. Monsieur Julien Doubrovsky, $1^{\mathrm{er}}$ prix de dissertation philosophique. Sur l'enveloppe décolorée, en lettres mauves. J'en suis encore bleu. Le grand amphi de la Sorbonne retentit des hymnes alliés. Mon père fou à lier, le mou déjà plus qu'à moitié bouffé aux mites, en larmes. Ma mère en transes, et tante Nénette et Riri, qui nous ont sauvé la vie, fiers comme si j'étais leur propre fils, ma sœur bien sûr, ma tante médaillée, mon oncle rescapé de Drancy, tous à l'appel, en bloc, debout, surgissent, quand on a entonné "La Marseillaise", aujourd'hui vous ne pouvez pas comprendre. Ma guerre, ma revanche, ça a été mon jour de gloire. Victoire personnelle. En juillet 45. Mais l'AUTRE. En mai. Pas la symbolique. La vraie. René Capitant, ministre de l'Éducation nationale, son discours encore résonne. J'ai encore le goût de son déjeuner, après, dans la bouche. Contre-filet rôti, haricots verts au beurre, bombe glacée, pour finir, tarte aux abricots, je la remâche comme une madeleine proustienne. Mais rien ne reparait autour. Je cherche le 8 mai. Je trouve mon second bac, en octobre avec mention bien. Pas fameux, mais ma santé n'est pas brillante. Je recense mes maladies. Tout l'été. Été entre la vie et la mort, spirochétose ictero-hémorragique, maladie rare, cinq ou six cas dans tout Paris, de la viande contaminée par les rats à la Villette. En fait d'abattoirs, je suis presque resté sur le carreau. J'ai intacte en tête 
la kyrielle de mes bobos mortels. Je ne retrouve toujours pas le 8 mai. Une date aussi prodigieuse. Une épiphanie de l'histoire. Je me replonge dans mes bas-fonds, jusqu'au tréfonds. Désespérément, je sonde, je fouille. Pas un reste, pas une trace. Tout a disparu dans un absolu naufrage. Comme une épave, je suis là, pétrifié, sur le terre-plein de l'Étoile. 8 MAI 45 : TROU DE MÉMOIRE ${ }^{40}$. "

\section{NOTES}

1. Voir le livre de Gérard Namer, Mémoire et société, Paris, Méridiens Klincksieck, 1987.

2. J. Laplanche \& J.-B. Pontalis, Vocabulaire de la psychanalyse, Paris, Presses universitaires de France, 1967, p. 427.

3. S. Freud définit ainsi les souvenirs écrans: «Nos souvenirs d'enfance nous montrent les premières années de notre vie, non comme elles étaient, mais comme elles sont apparues à des époques ultérieures d'évocation ; les souvenirs d'enfance n'ont pas émergé, comme on a coutume de le dire, à ces époques d'évocation, mais c'est alors qu'ils ont été formés et toute une série de motifs, dont la vérité historique est le dernier souci, ont influencé cette formation aussi bien que le choix des souvenirs» («Sur les souvenirs écrans», in Névrose, psychose et perversion, trad. fr. Paris, Presses universitaires de France, 1973, p. 113-132.).

4. P. Nora, ed., Les lieux de mémoire, Paris, Gallimard, 1984-1986, 3 vol. parus.

5. Voir le lumineux article de C. Ginzburg, «Signes, traces, pistes. Racines d'un paradigme de l'indice ", Le Débat, 6, 1980, p. 3-44.

6. Voir Eric Hobsbawm et Terence Ranger, eds, The Invention of Tradition, Cambridge-New York, Cambridge University Press, 1983.

7. M. de Certeau, L'écriture de l'histoire, Paris, Gallimard, 1975.

8. P. Ricœur, Temps et récit, Paris, Seuil, 1985, t. 1.

9. H. V. White, The Content of the Form. Narrative Discourse and Historical Representation, Baltimore, Johns Hopkins University Press, 1987.

10. R. Chartier, «Le passé composé », Traverses, 40, 1987, Théâtre de la mémoire, p. 9.

11. M. Halbwachs, La mémoire collective, Paris, Presses universitaires de France, 1968, rééd., p. 38-39.

12. D. Veillon, «La Seconde Guerre mondiale à travers les sources orales », Questions à l'histoire orale, Table ronde du 20 juin 1986, nº sp. de : Cahiers de l'IHTP, 4, 1987, p. 54.

13. Voir l'étude entre autres de Maurizio Catani, «Les migrants et leurs descendants entre devenir individuel et allégeance chtonienne ", Cahiers internationaux de Sociologie, LXXXI, 1986, p. 281-298.

14. Stendhal, «La vie de Henry Brulard », in Cuvres intimes, Paris, Gallimard, 1955, p. 147-148 (« Pléiade »).

15. M. Bakhtine, "Récit épique et roman ", in Esthétique et théorie du roman, trad. fr. Paris, Gallimard, 1978, p. 452.

16. À ce propos, je voudrais faire un certain nombre de précisions qui éclaireront je crois la portée de la discussion qui s'est établie depuis plusieurs années, à propos des récits de vie et/ou l'histoire orale. On me permettra de mettre le problème en place à l'aide de deux réflexions de M. Pollak: «... et en évitant aussi la tentation de transformer en une forme exclusivement littéraire l'approche biographique en sciences sociales ", écrit-il à propos de moi (M. Pollak avec Nathalie Heinich, «Le témoignage », in L'illusion biographique, ${ }^{\circ} \mathrm{sp}$. de : Actes de la Recherche en 
Sciences sociales, 62-63, 1986, p. 7) ; et : «... la vertu qu'attribue Régine Robin à la fiction réside dans la pluralité et dans l'ouverture des discours. La construction scientifique, par contre, tend à produire du discours fermé et clos, obsédée qu'elle est par le principe de cohérence. Ce reproche est autrement sérieux qu'une discussion qui s'arrêterait au seul style littéraire. Car comment décider pour les meilleurs travaux en sciences humaines qu'ils ne forment pas également un genre littéraire? Les enfants de Sanchez d'Oscar Lewis, illustre exemple de la méthode biographique en ethnologie, a toutes les qualités d'un roman familial, ce qui ne lui enlève rien de sa scientificité. De plus, cet écrit semble infirmer le procès fait par Régine Robin à l'écriture scientifique, à savoir d'être fermée et close, et donc insensible à la pluralité des voix et à l'ambiguïté de la réalité » (M. Pollak, « Pour un inventaire », in Questions à l'histoire orale, p. 24-25). La réponse un peu fournie à l'argument de M. Pollak demanderait de longs développements qui viendront en leur lieu et place. Je n'avais et je n'ai jamais opposé la fiction au discours scientifique (vieux débats), j'avais opposé la fiction dans ses plus riches potentialités aux récits de vie. Nuance. Et ici, en ce qui concerne mon propos, je dirais que c'est la fiction qui rendrait le mieux compte de cette mémoire culturelle (M4) dont j'ai parlé, de Proust à Musil, de Flaubert à Claude Simon. Je m'en explique, il est vrai trop rapidement, dans trois articles: R. Robin, «Enjeux : récit de vie, discours social et parole vraie ", Vingtième Siècle, 10, 1986, p. 103-110 ; «Frontières du fantasme ou récit du vécu? », Cahiers de Sémiotique textuelle, 8-9, 1986, p. 9-20 ; "L'histoire orale : quelques interrogations sur une pratique », in B. Jewsiewicki, ed., Récits de vie et mémoire : pour une anthropologie historique du souvenir, à paraitre.

17. F. Nietzsche, Considérations intempestives, cité par A. Finkielkraut, « Âge de l'idéologie, âge des cultures ", in J. Halpérin \& G. Lévitte, eds, Mémoire et histoire, données et débats, Actes du XXV Colloque des intellectuels juifs de langue française, Paris, Denoël, 1986, p. 110.

18. L. Passerini, « Work ideology and consensus under Italian fascism », History Workshop, 8, 1979.

19. Voir le livre récent d'A. Jaubert, Le Commissariat aux archives. Les photos qui falsifient l'histoire, Paris, Barrault, 1986.

20. R. C. Rowland, The Rhetoric of Menachem Begin. The Myth of Redemption through Return, Boston, University Press of America, 1985.

21. M. Begin, La révolte d'Israël, trad. fr. Paris, Plon, 1953 (The Revolt. Story of the Irgun, 1951) [NdE].

22. Voir en particulier Le fascisme dans son époque, trad. fr. Paris, Julliard, 1970, 3 vol.

23. J. Kocka, 23 septembre 1986, cité par Jean-Jacques Guinchard, "Passé nazi, passé allemand », Le Monde diplomatique, juil. 1987, p. 17.

24. L. Rebatet, Les décombres, Paris, Denoël, 1942 [NdE].

25. A. N. Rybakov, Les enfants de l'Arbat. Roman, trad. fr. Paris, A. Michel, 1988 [NdE].

26. T. Aïtmatov, Les rêves de la louve, trad. fr., Paris, Messidor, 1987, p. 239 (Plakha).

27. Voir le texte pionnier de D. Bertaux, Histoires de vies ou récit des pratiques?, Rapport au CORDES, $\mathrm{n}^{\circ}$ 23, mars 1976 ; et D. Bertaux, ed., Biography and Society. The Life History Approach in the Social Sciences, Beverly Hills, CA, Sage, 1981.

28. G. Lipovetsky, L'ère du vide, Paris, Gallimard, 1983.

29. C. Simon, « Discours à Stockholm », Lettre internationale, 8, 1986, p. 68.

30. M. Kundera, "Discours de Jérusalem: le roman et l'Europe", in L'art du roman, Paris, Gallimard, 1986, p. 198-199.

31. G. Scarpetta, L'impureté, Paris, Grasset, 1985, p. 307.

32. M. Kundera, « Discours de Jérusalem... », p. 199.

33. Je reprends ici l'essentiel d'un article que j'ai fait paraître dans une revue littéraire québécoise : R. Robin, « Le retour du lisible », Spirale, 68, 1987, p. 2-3.

34. D. Oster, Dans l'intervalle, Paris, POL, 1987, p. 102.

35. A. Robbe-Grillet, Le miroir qui revient, Paris, Minuit, 1984, p. 17.

36. Voir à ce sujet l'article stimulant de F. Ferrarroti, "Sur l'autonomie de la méthode biographique », in J. Duvignaud, ed., Sociologie de la connaissance, Paris, Payot, 1979, p. 131-152. 
37. L. Passerini, "Mémoire et histoire: la visite de Mussolini à l'usine Fiat Miraflori », Le Mouvement social, 16, 1984, p. 56.

38. Voir M.-F. Chanfrault-Duchet, «La doxa scolaire dans les récits de vie », Cahiers de Sémiotique textuelle, 4, 1985, p. 79-93.

39. S. Doubrovsky, « Trou de mémoire », Nota Bene, 17-18-19, 1986, p. 65-78.

40. Ibid., p. 77-78. 